\title{
Al-Based Decision System to Evaluate Scenarios of Response to the COVID-19 Pandemic. Evidence From Barcelona and Manhattan
}

Didier Grimaldi ( $\square$ didier.grimaldi@salle.url.edu )

University - La Salle, Sant Joan de La Salle

Hugo Arboleda

Universidad Icesi

Karla Esquivola

University - La Salle, Sant Joan de La Salle

\section{Research Article}

Keywords: COVID Pandemic, Artificial Intelligence, Decision Support System, Small and Medium Business, Geographical Information System

Posted Date: December 20th, 2021

DOI: https://doi.org/10.21203/rs.3.rs-1172480/v1

License: (c) (i) This work is licensed under a Creative Commons Attribution 4.0 International License. Read Full License 
Title: Al-based decision system to evaluate scenarios of response to the COVID-19 pandemic. Evidence from Barcelona and Manhattan.

\section{$\underline{\text { Author names and affiliations: }}$}

- Dr. Didier Grimaldi. Ramon Llull University - La Salle. Sant Joan de La Salle, 4208022 Barcelona. Spain. didier.grimaldi@salle.url.edu

- Dr. Hugo Arboleda. Director of the Master in Project Management. Director of the Master in IT Management. Universidad Icesi. Calle 18 no. 122-135 Pance, Cali, Colombia. hfarboleda@icesi.edu.co

- Karla Angélica Esquivel Huitrón, Master in Data Science. La Salle Campus. Ramon Llull University - La Salle. Sant Joan de La Salle, 4208022 Barcelona. Spain.karla.eh11@gmail.com

\section{Corresponding author:}

Dr. Didier Grimaldi didier.grimaldi@salle.url.edu

Keywords: COVID Pandemic; Artificial Intelligence; Decision Support System; Small and Medium Business; Geographical Information System 


\section{Abstract}

In 2020, the COVID-19 outbreak has had severe economic and social consequences all over the planet. Traditional national health systems have been unable to predict and to provide a decision support system able to coordinate an effective response to the outbreak and to stop the rapid spread of this disease. In this current manuscript, we have decided to focus our analysis on Small and Medium Businesses (SMBs). While some SMBs have survived, many others, particularly in urban areas, have had to shut down as a direct decision of government restrictions. Our study presents a decision support system based on Artificial Intelligence which helps governments to prioritize the closures of SMBs located in a city. Indeed, the decision to shut down may vary according to the relative danger that the business premises represent as a social gathering point and its benefits for the local economy. In this vein, we analyse 3 different scenarios which assume different financial and social costs. The visualization of the results on a city map provides additional value for the decision-making process. The Urban Decision Support System is tested by two case studies: Barcelona and New York City. This research has implications for practitioners to support their decision to close-down the economies in the event of another large-scale outbreak. It has also research implications as new evidence that data analytics could be an additional and valuable source of information for decision support processes.

\section{Introduction}

\subsection{Background}

Globalization, the process by which national economies have immersed into a large interconnected global economic system has recently shown a new and substantial threat: the rapid diffusion of a global pandemic. With more than 62 million people infected and around 1.5 million deaths, at the time of writing this study [1], COVID-19 ('CO' stands for corona, 'VI' for virus, and 'D' for disease) has been the most lethal virus in recent history [2]. The 
outbreak was first reported in Wuhan, China a very dynamic industrial and commercial city, then it exponentially spread around the globe. Government response was generally chaotic and improvised in the majority of cases since the outbreak caught everyone off guard. Likewise, scientists were neither able to explain the fast propagation of the virus that was suddenly threatening the health of millions of people in a very short time [3], nor come up with actions to prevent it, or to predict a date when a vaccine would be available.

The only solution, adopted by the vast majority of countries, was to impose a blanket lockdown which mean confining citizens to their homes and stopping letting people go to work or schools, stopping public transport and isolating the country by closing national boundaries. It is estimated that global trade will fall by $30 \%$ in 2020 [4]. Small and Medium Business (SMB), which represents the heart of the economy for most countries, takes the worst hit in terms of jobs loss [5-7]. It will be consequently the scope of our study. Scientists all over the world engaged in a tremendous race to find not only the vaccine but also solutions to mitigate the dramatic symptoms of this disease (high respiratory distress, loss of smell and taste, high fever, etc.). The analysis of these events shows there is a need to develop effective decision systems to fight epidemics and then monitor the actions which have been taken. Indeed, depending on the response strategy, the consequences of COVID19 will be different in terms of social costs i.e. the number of infected, and financial ones, i.e. the number of SMBs shut-down.

This current pandemic has shown that the national health surveillance systems were not efficient enough to alert the authorities and to help them to take well-informed decisions. This is a gap that methods employing Artificial Intelligence (AI) could cover. Data analytics have already shown their potential in past citizen health protection strategies, although these methods have not been fully embraced by authorities. Ginsberg et al. [8] has 
demonstrated that listening to health-seeking Google queries is a good marker to detect pandemics. Gerber [9] adds that Twitter can be used to identify urban insecurity and potential physical risks. The application of semantic analysis [10] demonstrates how Al could be an aid for crime prediction in the city of Chicago (Illinois, USA), a complementary solution to the standard approach of policy patrolling. So, even the few scientific studies that exist show that there is still a gap between the expectations provided by the millions of data generated every day and their potential use to improve citizens' life and especially their health protection.

\subsection{Our focus and contribution}

Our paper responds to this lack of scientific and academic input within the field of Decision Support System (DSS) to respond to a pandemic outbreak. Our study asks the following research question: to propose an Urban DSS (UDSS) which helps governments to prioritize closures of SMBs in order to respond to a pandemic outbreak according to the financial and social costs they are prepared to assume. The scenarios may indeed vary according to the equilibrium point decided between economic and social aspects. Through the use of 4 Machine Learning unsupervised techniques, the UDSS responds to three different scenarios (a) reducing social costs to the minimum but assuming high financial costs, (b) medium impact in terms of social costs and financial costs and (c) reducing financial costs to the minimum but assuming high social costs. By obtaining information on the consequences of each scenario, the authors believe the governments should be able to take a more efficient, robust and transparent approach to pandemics. This study contributes to decision making for pandemics by covering the 3 following research objectives:

- the identification of new types and sources of information (geographical, epidemiological, consumer behaviour...), 
- demonstrate how this mix of types and sources could have an effective impact in managing uncertainty during a global pandemic,

- a comparison of diverse Al algorithms in terms of their effectiveness for pandemics response.

\subsection{Structure of the paper}

The remainder of this manuscript is organized as follows. In the next section, we analyse the literature related to the COVID-19 impact and responses. In section 3, we describe the different sources of data collected. In section 4, we develop the model and determine the best algorithm to support the UDSS and respond to the research question and objectives. In section 5, we present the results concerning the cities of Barcelona (Spain) and New York City (USA). The next section discusses the results that are obtained and presents the implications for practitioners and future research. The last section is a short conclusion.

\section{Literature Review}

We respectively cover in this section the financial (2.1), commercial (2.2), business (2-3) and working factors (2.4) related to the UDSS developed in the section 4. History has been marked by the fear for pandemic diseases and the consequent need to find effective solutions to respond to them. The emergence of bioinformatic technologies has increased our understanding of biological phenomena by observing the viral process involved in the molecular infection. Outbreaks are the result of several biological phenomena events. Indeed, the growing global population and the increasing alimentary interaction between people and animals favour the emergence of new microbial threats and the transfer of new viruses to human being. Pandemics are becoming increasingly frequent [11]. The detection of the outbreaks is quite difficult. The more sophisticated health surveillance systems (e.g. USA and European) are based on weekly virologic and clinical surveillance which need 1 full week to collect the necessary data. Consequently, they can take up to 2 weeks to give the 
warning. However, non-conventional methods employing Machine Learning techniques have been recently tested to improve the speed of detection [12].

Machine Learning is a subset of Artificial Intelligence which has mainly 2 modes of running: supervised and unsupervised. In the first case, it employs statistical algorithms to train machines from a labelled dataset. The systems then look for optimizing models that best explain new and unknown data. In the second mode, unsupervised learning looks for possible patterns in an unlabelled data set and without human supervision. One of its main methods is the cluster analysis that the authors use in this study. Machine Learning unsupervised experimentation has been already carried out for pandemics surveillance. Indeed, Ginsberg et al. [8] train a system of user-generated queries placed in Google with the objective of detecting seasonal influenza epidemics. Due to the daily frequency of analysis, they claim their results are immediate compared to the national health system and can therefore provide an additional and valuable source of information for the early detection of virus propagation.

Medina [3] argues that it is practically impossible to prevent pandemic outbreaks. Indeed, we are living in a globalized and interconnected world where the tourism industry has been skyrocketing for the last 10 years. Regular flights are proposed for tourists to travel in few hours from the inner centre of a high-crowded city (such as New York, Paris, London) to rural zones where health conditions are very poor, such as Wuhan (capital of Hubei province in Central China). The COVID 19 was propagated in this poor Chinese rural zone due to the absence of basic health-care coverage and the delays in the detection of the virus. The passenger of a weekly flight between Wuhan and Milan [2] was later infected and this may well have been the beginning of the global spread of the disease. Nevertheless, it is worth noting other pandemic viruses such as: SARS (Severe Acute Respiratory Syndrome), Malaria, 
avian flu were propagated from other regions of the globe (e.g. South Asia, Russia, Congo, Ivory Coast) but only had a regional scope. Commercial packages form part of today's overinterconnectedness. Low-cost airlines increase this mix of world population by offering return tickets to global capitals every weekend and as a consequence infectious diseases and contamination are constantly diffused all over the planet in 48 hours.

\subsection{Economic impact of business shutdown}

In the peak of the COVID-19 pandemic outbreak, the governments found it very difficult to estimate the long-term effects at business and societal levels. But most of them has considered that our lives and safety prevailed before efficiency and economic profit. Accordingly, they took the decision to shut down most of the industries and services including global supply chains [13], manufacturing[14], retail services [15], tourism [16] and education centres. Our societies which were widely open systems that imported most of the products that we eat, or wear were asked to close their national boundaries. In this vein, people were compelled to stay at home all day. Many democratic governments had not imposed such severe restrictions on individuals' freedom since World War II.

Local SMBs providing non-primary necessity (food, cleaning or care treatments) were obliged to physically close. Consequently, new habits emerged making the store coming to home instead of the consumer going to the store [17]. As an example, in the United Kingdom online commerce grew exponentially [5]. If the governments' decision to close these shops was generally accepted by citizens with little opposition, the consequences are currently substantial, and we are now beginning to wonder if other scenarios existed. In other words, would we be in a better or worse situation if different decisions had been taken?

But are we able to estimate, given a scenario of shutdown, how many SMBs will close? What will the remaining occupancy rate be? These questions are particularly important for SMBs, 
which for their so-called 'liability of smallness' were the largest sector affected by the COVID-19 [18]. This study fills this gap. The current scenario points out that economic growth in China will drop to 3.2\% [4] and the US economy is expected to fall by $2.9 \%$ in 2020. Spain, Greece and Portugal stand to lose 10\% of their GDP in 2020 due to their high dependence on service and tourism industries [4]. At the same time, the number of deaths caused by COVID-19 has reached 1.5 million [1]. This unprecedented scenario has hit governments hard, and most have lacked a clear strategy to deal with both health and economic issues, improvising solutions as they went along.

\subsection{Customer preferences}

Consumers develop habits and preferences over time about what to consume, and when and where to do so [17]. They have preferences about the categories of the products they buy, in other words, priority for them. Customer behaviour, needs or preferences change between a normal situation (Pre-COVID), a confinement or a Post-COVID new reality. They are indeed location and time bound. During periods of uncertainty, people usually postpone purchasing non-priority products i.e. durable goods (automobiles or household appliances) and discretionary services (e.g. theatres, operas, sports, movies, bars or restaurants) [17].

As a consequence, some SMBs have had to close down due to their lack of understanding of the new habits from their regular customers [19]. They were unable to pivot and propose alternate ways of serving their existing clients [15]. Others localized in the same commercial street of a district have perceived the changes of behaviour and decided to mutualize their effort. Financially supported by the economic department of their city, they have developed a joint ecommerce platform to digitalize their point of sale and propose their products 
online in a double-sided local marketplace (such as eBay or Amazon). Dinan (a small town in the West France) and Barcelona are just two examples ${ }^{12}$.

\subsection{Essential versus non-essential products or services}

The authorities defined what essential versus non-essential products and services are. Essentials are maintained when physical contact between client and employee (i.e. pharmacy, supermarket) is required, while non-essentials have to be digitalized if they want to keep running [13]. This definition slightly varied according to the country, government or even state in the same country (California versus New York in USA). For instance, in Spain hairdressing was considered as essential while France it was not [15]. It shows the lack of coordination between countries and especially those that constitute the European Union. This absence of a common definition also resides in the difficulty to understand the messages sent by the World Health Organization (WHO from now). For instance, in March the WHO affirmed that the COVID risk was not associated to an aerosol-related transmission and the risk of propagation was low through a conditioning air system. 3 weeks later, they affirmed the contrary. Besides, the distinction has varied depending on the urban-rural proportion of the country generating further confusion. Indeed, many activities are considered as essential in rural zones, where population density and human contact, the main form of contamination, is inferior[18].

\subsection{Working conditions and the risk of COVID transmission}

An analysis of working conditions for SMB, carried out by Kristof [20], states the personenvironment fit (coined as the 'P-E' fit) which measures the adherence by the employee of

\footnotetext{
${ }^{1}$ https://dinan.maville.com/actu/actudet -quimper.-un-site-web-j-aime-mes-commerces-va-etre-creepour-aider-le-commerce-local fil-4362790 actu.Htm - last access on 01/12/2020

${ }^{2}$ https://ajuntament.barcelona.cat/noubarris/pla-desenvolupament-economic/ca/noticia/lajuntamentimpulsa-una-bateria-de-mesures-per-fomentar-la-digitalitzacio-dels-comercos-i-la-restauracio-debarcelona-2-2-2-2 964117 - last access on 01/12/2020
}

Page: 9 
the organization values, cultures and work conditions of the employer has large implications on the workforce productivity. SMBs do not impose the same working conditions and the same safety measures on their staffs as on their clients [17]. Therefore, the risk of a worker being infected by the COVID varies depending on the category and the sector of activities [15].

As far as working conditions are concerned, the most important risk factors are the distances maintained between the customer and the worker and the type of interaction (Face-to-Face, telephone, 1-meter physical distance, use of protective window). For instance, a beautician has closer, longer and riskier interaction than a public employee responding to a citizen's query over the phone. All SMBs have introduced contactless payments for any amount of sales and made great efforts to improve their range of e-commerce products and home delivery[17]. Some businesses have facilitated home-working for all of their employees, while others have decided to have $50 \%$ of their personnel at home while the other $50 \%$ carry out tasks from their usual office[16].

In a nutshell, the tremendous speed of contamination and the high mortality rate of the recent pandemic have highlighted how completely unprepared for largescale outbreaks public institutions were, and possibly still are. Determining a scenario of responses which are efficient enough to slow down the virus propagation while limiting also risk of closure for SMBs will be the challenge for future governments and public institutions. Our study aims at covering this gap by developing an Urban Decision Support System (UDSS) which determines if each business in a given location should be kept open or closed according to the scenario decided. We consider 3 different scenarios which are strongly influenced by economic and social scales of cost. They represent the type of decision that many governments had to take to answer to this pandemic. Through the use of unsupervised 
Machine Learning techniques, the authors wish to offer a complementary solution to the existing traditional methods in use.

\section{Data Collection}

In December 2020, Spain recorded the third-highest coronavirus fatality rate in Europe. Official figures state there have been 345,563 confirmed Covid-19 cases. Barcelona is a city on the north-eastern coast of Spain. With a population of 1.6 million, it is the second most populated municipality of Spain (after Madrid, the capital) and the rate of COVID mortality and hospitalized people had been relatively high throughout the outbreak [1]. The UDSS model requires 3 datasets:

- The urban localization and categorisation of all the commercial locations are provided by the observatory Eixos (https://eixos.cat/html/portada-1-eng.html) which realises audit and consulting services and resolves urban management issues (transport, housing, economic...) for many cities in the world. The author uses their dataset which includes 19 attributes for each location: Name, GPS coordinates, district, neighbourhood, category of the business, labour occupation rate i.e. the average number of workers per square meters[21].

- The results of a survey on citizens located in Barcelona conducted from May $13^{\text {th }}$ to June $1^{\text {st }}, 2020$ during the COVID-19 outbreak. Each interviewee was asked to answer 30 discrete choice experiments by choosing amongst 2 options which type of location he would prefer to be re-opened. We have included the experiment instructions, a sample of the questionnaire and the demographic distribution of the answers in Appendix A. This type of experiment, called single binary discrete choice, predicts real behaviour quite accurately [22]. In total, 617 people responded, and 18,510 decisions were obtained. The questionnaire includes an optional field in case the interviewees want to 
receive the conclusions of this present research. The figure 1 shows the results of this survey classifying the user preferences by SMB category.

- We collected the personal opinions of different groups of epidemiologists and infectious disease specialists (more than 600) reported in many newspapers such New York Times [23], Business Insider [24], Reuters [25] and others [26,27] during May and June 2020. Interviewees were asked to classify the contamination risk level depending on the SMB business category.

Figure 1: User preferences by business category

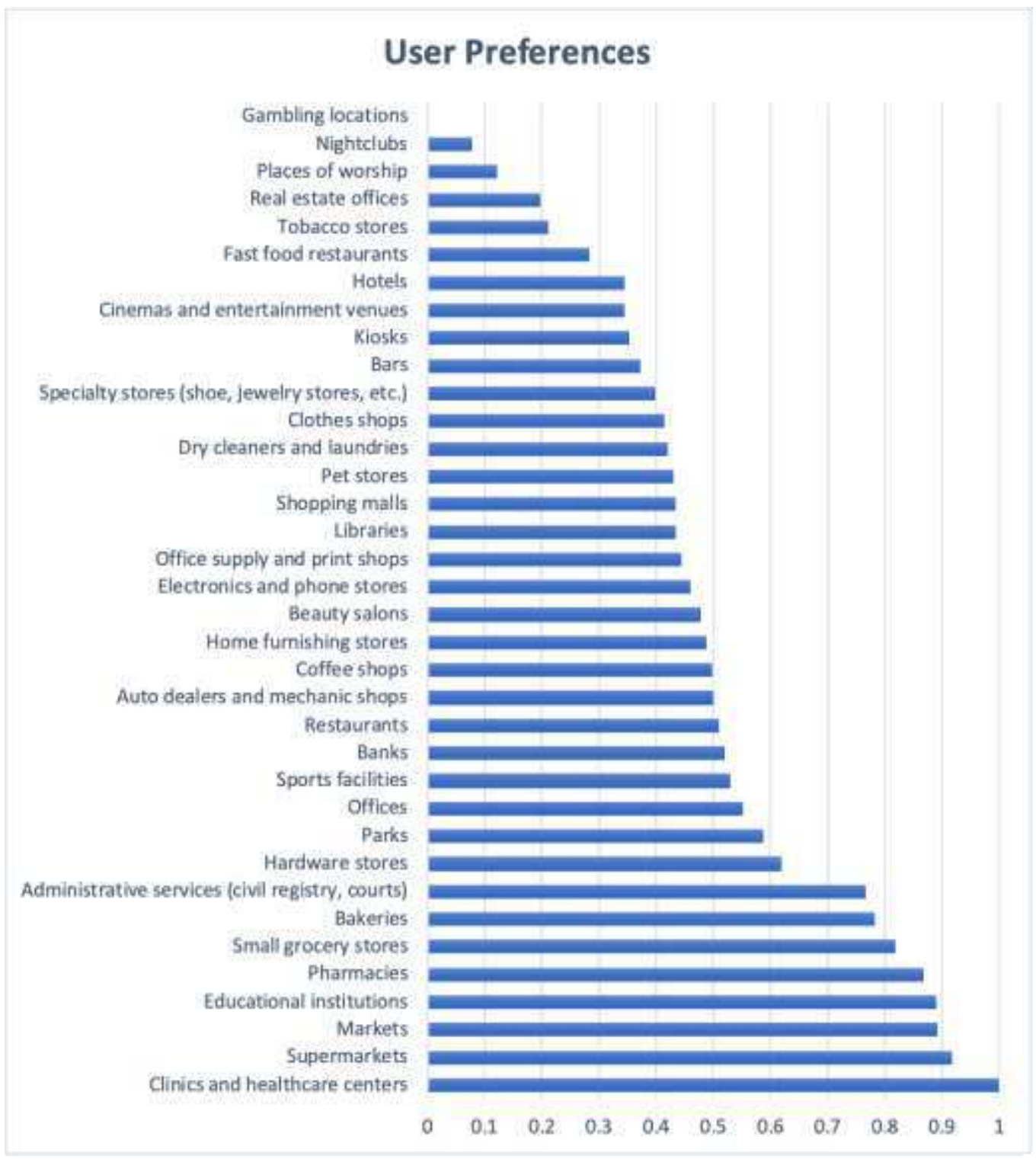




\section{Model development}

This section develops our model.

\subsection{Problem statement}

The UDSS helps decide whether each SMB location should stay open or closed to achieve the objectives defined by a scenario of response. The UDSS returns a table which contains all the locations labelled with a status either "open" or "closed" (table 3). The scenarios are: (a) optimizing social costs, (b) optimizing financial costs (c) a trade-off between them. The model is developed using the case of Barcelona and its inputs are geographical, epidemiologist and opinions (users' preferences). The UDSS has a visual module to map the results in a city map (figures 5 and 6).

\subsection{Analytical approach}

Let's consider a SMB as a location $L(p)$ geo-positioned at a spatial point $p=$ (longitude, latitude) in the city map. For each location $L(p)$, we define the vector $V(p)$ (equation 1 ) with the 4 following features: $f 1(p), f 2(p), f 3(p)$ and $f 4(p)$. The feature $f 1(p)$ quantifies the users' preference votes based on the web-based survey (Appendix A and figure 1). For each location, the feature $f 2(p)$ points out the type of product or service and whether it is considered essential or not. It is based on the classification generated by the Eixos observatory. In the next subsections 4.3 and 4.4 , we present features $f 3(p)$ and $f 4(p)$ which respectively quantify the financial risk and the social transmission risk of a location $L(p)$.

$V(p)=[f 1(p), f 2(p), f 3(p), f 4(p)]$

Using these 4 features as predictors, the authors use (section 4.5 ) and benchmark 4 clustering algorithms (section 4.7) to define clusters of SMBs. The best clustering method (section 5.2) is applied to compute the UDSS (section 5.3) and calculate the results (section 
5.4). The three possible scenarios correspond to: (a) optimizing social costs -> by closing all the clusters except those with essential products; (b) optimizing financial costs -> by keeping all the clusters opened except those with high transmission COVID risk, (c) a trade-off -> only high-preference categories are opened. The graphical module of the UDSS provides a visualization of the results on the city map (section 5.5).

The UDSS classifies first the locations into $k$ clusters using the vector of predictors $V(p)$ (equation 2). Then, it returns for each Cluster (i) its status "Opened" or "Closed" depending on the considered scenario (equation 3):

Cluster $(\mathrm{i})=\sum_{p(i)}[L(p), \mathrm{V}(\mathrm{p})]$

with $1 \leq \mathrm{i} \leq \mathrm{k}$

UDSS $[\operatorname{scenario}(j)$, Cluster(i)] = "Opened" or "Closed".

with $1 \leq \mathrm{j} \leq 3$

with $1 \leq \mathrm{i} \leq \mathrm{k}$

In words, Equation (2) determines the i-partition of locations $L(p)$ geo-localized with its $p$ (longitude and latitude) and with the 4 features $V(p)$ form the cluster i. Equation ( 3 ) determines that the UDSS returns for each of the k Clusters the label "Opened" or "Closed" according to the chosen scenario $\mathrm{j}$.

The whole UDSS with its different modules and inputs are shown in figure 2.

Figure 2: UDSS framework
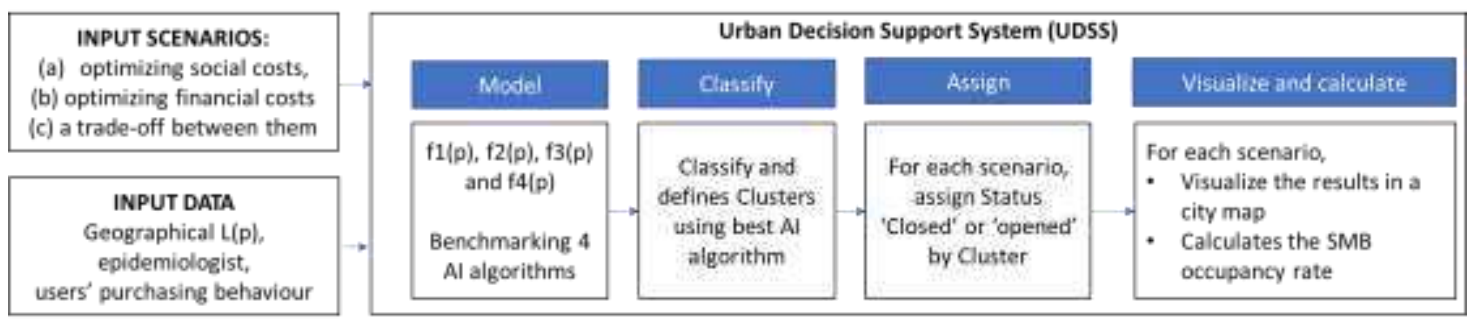


\subsection{Feature $\mathrm{f3}(\mathrm{p})$ : financial risk of a location}

The EIXOS observatory calculates and analyses the Commercial Attraction Index (CAI) of a location based on its geographical position in the city map [21]. In complex network theory [28-30], a network is made up of a set of nodes connected by links, although not all nodes are equally important since they are usually affected by a different number of connections. We apply this theory: in our case, nodes correspond to locations and links are streets joining the nodes or are 'cul-de-sacs'. The CAI is related to the betweenness concept which estimates the flow of clients by determining how many routes each point goes through on the network [21]. The CAl index is highly correlated with the financial risk of a location since displacement becomes very limited during a pandemic crisis. Mathematically, the CAI index is the sum of geodesics that pass through a location $L(p)$. It is calculated by the equation (4):

$\operatorname{CAI}(L(p))=\sum_{y \in N} \sum_{z \in R y} O D(y, z, L(p)) \frac{W(z)}{\text { total weight }(y)}$

where total weight $(y)$ is the total weight in radius from each $y$. In this formula, $y$ and $z$ are origin and destination points respectively. Weighted by the proportion of network quantity accessible $\mathrm{W}(\mathrm{z})$ from geodesic origin y that is represented by geodesic destination $(\mathrm{z})$.

A SMB which needs a high employment rate (i.e. number of workers per square meters) also suffers a high financial risk. Therefore, the authors consider the financial risk $f 3(p)$ as the arithmetic mean of the CAI and the employment rate. In other words, this feature assumes a greater intensity of workforce and a lower percentage of geographical commercial attraction, leading to a high financial burden and a high risk of collapse and bankruptcy.

\subsection{Feature $f 4(p)$ : COVID transmission risk}

This feature $\mathrm{f} 4(\mathrm{p})$ calculates the COVID transmission risk inside the premises of a location $L(p)$. This risk is a variable of each business category. We decide to classify activities: as low risk (1-3) according to what epidemiologists [23] would do early, as medium risk (4-7) next 
year, as maximum risk ( 8 to 10 ) one year or more. To differentiate the activities within each business category, we have used other studies [24-27,31]. Once we have the list of activities with the risk assigned from 1 to 10 , we obtain values with a range from 0 to 1 with the following formula (5):

$\mathrm{f} 4(\mathrm{p})=\frac{R(p)-\min }{\max -\min }$

\subsection{Algorithms used}

We present in this sub-section the 4 algorithms applied and benchmarked: k-Means, Balanced Iterative Reducing and Clustering using Hierarchies (BIRCH from now), Agglomerative and Density-based spatial clustering of applications with noise (DBSCAN). These choices correspond to 3 classes of method that we wished to test:

- Partitioning methods: grouping techniques that subdivide the data sets into a set of $\mathrm{k}$ groups, where $\mathrm{k}$ is the previously specified number of groups. In our study, we employ the k-Means partitioning.

- Hierarchical clustering: clustering techniques that subdivide data sets and result in a tree-based representation of objects. We use the $\mathrm{BIRCH}$ and Agglomerative methods.

- Density-based clustering: clustering techniques based on the general idea that a group in a data space is a contiguous region of high-point density, separated from the other groups by contiguous regions of low-point density. We use DBSCAN.

\subsection{1 k-Means}

K-Means is an unsupervised learning algorithm that groups the data set into different clusters. The algorithm follows these steps:

- Start with a first group of randomly selected centroids, which are used as starting points for each group. 
- Iteratively compute records to clusters and adjust the cluster centres to optimize their positions [32].

- The optimization is stopped when the centroids have stabilized, i.e., the clusters can't improve refinement, or the established number of iterations has been reached. The optimization heuristic calculates the sum of the squared error i.e. the sum of the squared distance between the data points and the cluster centroid (in other terms, the arithmetic means of all data points belonging to that group). The less variation there is within the clusters, the more homogeneous (similar) the data points are within the cluster.

The major disadvantage of this algorithm is that it assumes that all sets are spherical, and the number of clusters needs to be set (section 4.6).

\subsubsection{BIRCH (Balanced iterative reducing and clustering using hierarchies)}

$\mathrm{BIRCH}$ is an algorithm used to perform hierarchical clustering on particularly large data sets. It uses a tree structure to create a cluster, and each node in this tree is made up of various clustering features. The algorithm works as follows:

- Start with an initial threshold value, scan the data, and insert points into the tree.

- Filter the tree created in the first step to remove some abnormal nodes (optional).

- Group all the tuples to get a better tree (optional).

The main point of this algorithm is the first step, where the tree is generated, and the other optional steps are to optimize the result. In most cases, $\mathrm{BIRCH}$ only requires a single scan of the database. Its main advantage is that computation is fast [33]. However, the data pooling is not efficient and may be slow with large datasets. 


\subsubsection{Agglomerative}

Agglomerative is a bottom-up approach, i.e. each observation starts in its own group and the pairs of groups merge as they move up through the hierarchy. The algorithm works as follows [34]:

- Begin by treating each object as a single cluster.

- Determine the measure of distance and calculate the distance matrix.

- The pairs are then merged successively until all the groups are merged into one large group containing all the objects.

- The result is a tree-based representation of the objects.

\subsubsection{DBSCAN (Density-based spatial clustering of application with noise)}

DBSCAN is able to find groups of arbitrary shapes and groups with noise [35]. The algorithm works as follows:

- The algorithm proceeds by arbitrarily choosing a point in the data set (until all points have been visited).

- Then it finds the points within a radius of $\varepsilon$ (eps) and identifies the centre of the points with the maximum neighbouring points.

- The groups are then expanded by recursively repeating the neighbourhood calculation for each neighbouring point.

One of the main advantages is it cannot properly group data sets with large density differences.

\subsection{Hyper-parameter settings}

For the k-means algorithm, the parameter $\mathrm{k}$ is calculated applying the Elbow method. This heuristic method of interpretation and validation of consistency is designed to find the appropriate number of segments in a data set. It consists of plotting the explained variation 
as a function of the number of groups. $\mathrm{K}$ corresponds to the point where the marginal gain decreases i.e., the elbow of the curve [36].

\subsection{Evaluation criteria}

To evaluate the results of each algorithm, three indexes are compared: Silhouette coefficient, Calinski-Harabasz, and Davies-Bouldin Indexes [37]:

- Silhouette coefficient: this is a method of interpretation and validation of coherence within the data groups. It calculates a measure of how close each point is in a group of points to the neighbouring groups between a range of $[-1,1] .1$ means that the points are very close to their own group and far from other groups, while -1 indicates that the points are close to neighbouring groups [38].

- Calinski-Harabasz index also known as the variance ratio criterion. The higher its score, the better the results since this means the clusters are dense and well separated[38]. This index is defined as the ratio between the average dispersion between clusters and the dispersion within the cluster. The equation (6) expresses it:

$$
s=\frac{\operatorname{tr}\left(B_{k}\right)}{\operatorname{tr}\left(W_{k}\right)} \times \frac{n_{E}-k}{k-1}
$$

Where $\operatorname{tr}\left(B_{k}\right)$ is the trace of the dispersion matrix between groups and $\operatorname{tr}\left(W_{k}\right)$ is the trace of the within-cluster dispersion matrix defined by equations (7) and (8):

$$
\begin{aligned}
& W_{k}=\sum_{q=1}^{k} \sum_{x \in C_{q}}^{T}\left(x-c_{q}\right)\left(x-c_{q}\right)^{T} \\
& B_{k}=\sum_{q=1}^{k} n_{q}\left(c_{q}-c_{E}\right)\left(c_{q}-c_{E}\right)^{T}
\end{aligned}
$$

$C_{q}$ is the set of points in the cluster $q, c_{q}$ is the centre of the cluster $q, c_{E}$ is the centre of the dataset and $n_{q}$ is the number of points in the cluster $q$.

- The Davies-Bouldin index is the average "similarity" between groups, where similarity is 
a measure that compares the Euclidian distance between groups with the size of the groups themselves [38]. A lower Davies-Bouldin index is related to a model with better separation between clusters, with 0 being the lowest score. Its formula (9) is

$$
D B=\frac{1}{k} \sum_{i=1}^{k} \max _{i \neq j} R_{i j}
$$

where the similarity is $R_{i j}$

\section{Evaluation and results}

The programming language used for the computation is Python. The libraries used are Matplotlib and Seaborn for data visualization, Numpy and Pandas for data management and analysis, SciKit-Learn for clustering algorithms. The visualization of the results is done with the Quantum GIS (QGIS) open-source software platform (https://www.qgis.org/en/site/ ).

\subsection{Transmission risk by category}

We calculate the transmission risk by category of activities (figure 3 ). 
Figure 3: COVID risk by business category

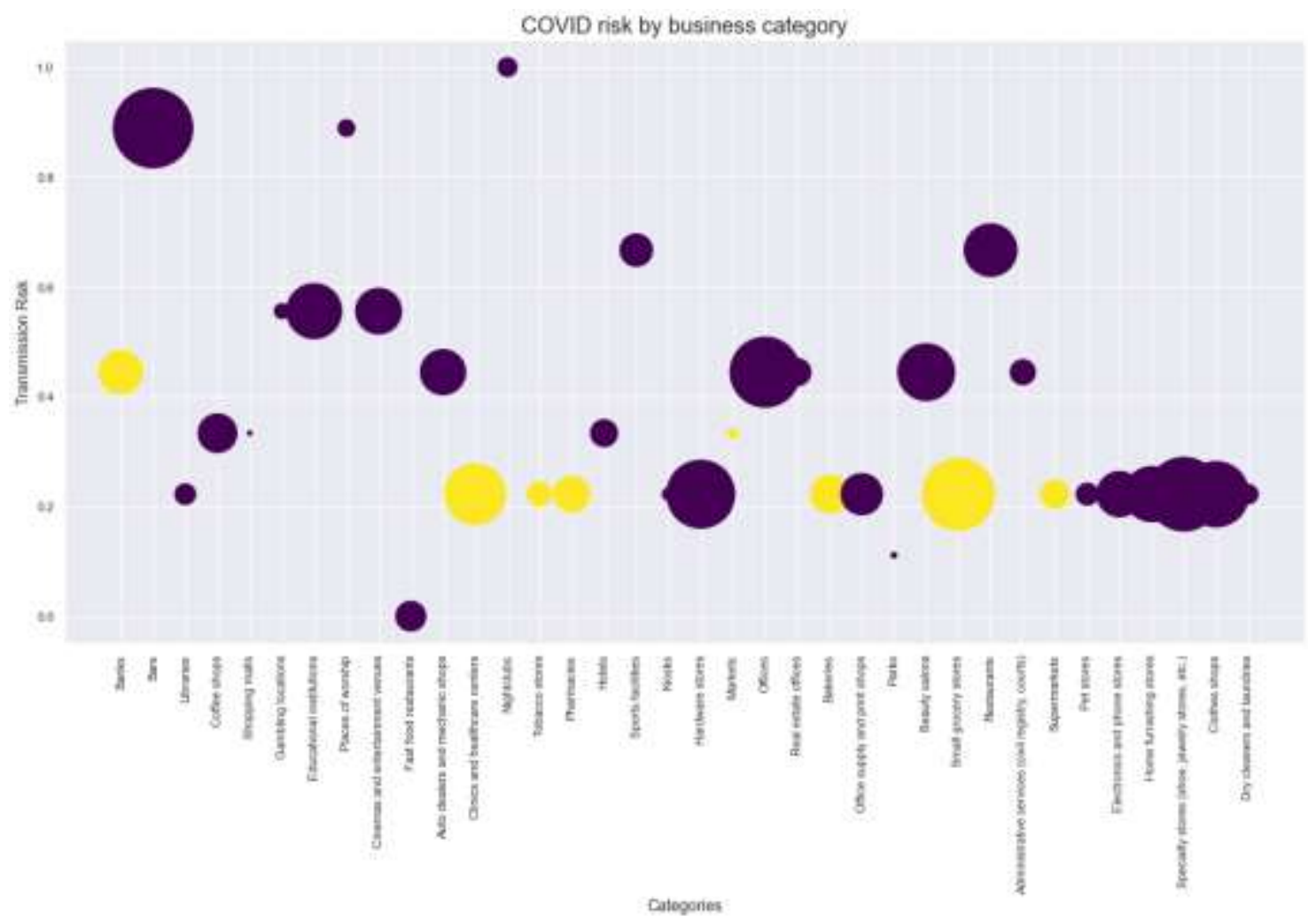

\subsection{Defining the hyper-setting $\mathrm{k}$}

The optimal number is set at 4 for the k-Means according to Elbow Curve (figure 4).

Figure 4: Elbow curve

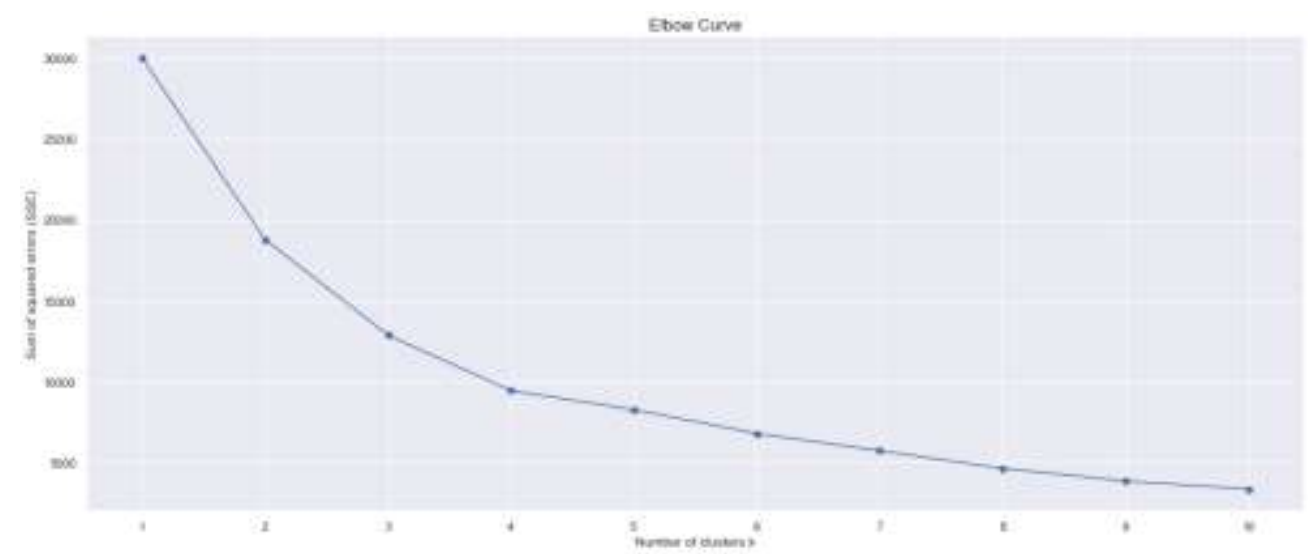

5.2 Evaluation benchmark of the four algorithms

An evaluation benchmark of the four algorithms is done. The results are in the table 1. 
Table 1: Algorithms benchmark results

Algorithm Silhouette Coefficient Calinski-Harabasz Index Davies-Bouldin Index

\begin{tabular}{rrrrr}
\hline 0 & KMeans & 0.457042 & 40449.536820 & 0.893715 \\
1 & BIRCH & 0.468613 & 38425.611431 & 0.839348 \\
2 & Agglomerative & 0.487798 & 20888.670738 & 0.730178 \\
3 & DBSCAN & 0.487798 & 20888.670738 & 0.730178
\end{tabular}

According to the Silhouette Coefficient and the Callinski - Harabasz Index, the algorithm that generates the most separated and dense clusters is k-Means. Therefore, we use it to compute the UDSS model.

\subsection{Computing the UDSS model using best clustering solution}

K-Means defines the following 4 clusters as shown in the table 2 .

Table 2: Clustering of the locations

\begin{tabular}{|c|c|}
\hline Cluster & \multicolumn{1}{|c|}{ Description } \\
\hline Cluster 0 & $\begin{array}{r}\text { Low preference, high transmission risk, high financial } \\
\text { risk, with no products and nonessential }\end{array}$ \\
\hline Cluster 1 & $\begin{array}{r}\text { Low preference, medium transmission risk, low financial } \\
\text { risk, with products and nonessential }\end{array}$ \\
\hline Cluster 2 & risk, with products and essential \\
\hline Cluster 3 & High preference, medium transmission risk, low financial \\
& risk, no products and nonessential \\
\hline
\end{tabular}


For cluster 0 , the most repetitive sectors are automobile dealers, car workshops, bars, clothing stores, restaurants, coffee shops, fast food, hotels and hairdressers and aesthetics. In cluster 1 , the most frequent sectors are: specialty shops, offices, building materials and hardware stores, home equipment stores and household equipment stores. For cluster 2, the most frequent sectors are small food stores or supermarkets, health care centres and medical clinics, bakeries and pharmacies. Finally, in cluster 3 the main sectors are banks, sports facilities, administrative services, educational centres, bookstores and libraries.

\subsection{Results of the study}

The results of the study by scenario are (table 3):

- Scenario 1 (social): reducing social costs to the minimum. High financial costs. According to UDSS, the decision is only essential activities are opened i.e. Cluster 2.

- Scenario 2 (trade-off): medium impact in terms of social costs and financial costs. Consequently, the decision is only high-preference activities are open, i.e. Clusters 2 and 3.

- Scenario 3 (financial): reducing financial costs to the minimum. Consequently, the decision is only Cluster 1 is closed because it is the cluster with the best alternative solution of tele-working and 'store-to-home' solutions.

Table 3: Results by scenario

\begin{tabular}{|c|c|c|c|c|}
\hline UDSS answer for each scenario & Cluster 1 & Cluster 2 & Cluster 3 & Cluster 4 \\
\hline 1- Social & Closed & Open & Closed & Closed \\
\hline 2- Trade-off & Closed & Open & Open & Closed \\
\hline 3- Financial & Closed & Open & Open & Open \\
\hline
\end{tabular}




\subsection{Visualization of the results}

Based on the UDSS results, the authors visualize and calculate the SMBs occupancy rates for each scenario. In a pre-quarantine and standard scenario, the average SMBs occupancy of Barcelona was $83 \%$. In scenario 1 , the average SMBs occupancy is $12 \%$. In the scenario 2 , the average SMBs occupancy is $45 \%$ and in the scenario 3, the average SMBs occupancy is $69 \%$. The UDSS has a visualization module. It shows the SMB occupancy rate for each of the 20 Barcelona districts (figure 5).

Figure 5: Visualization of the different scenarios of Barcelona close-down

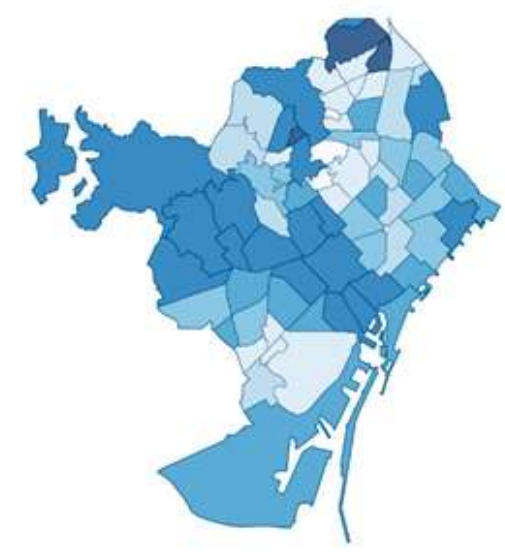

Pre-quarantine

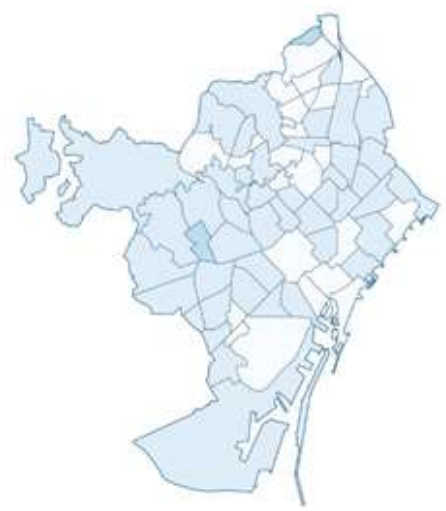

Scenario 2

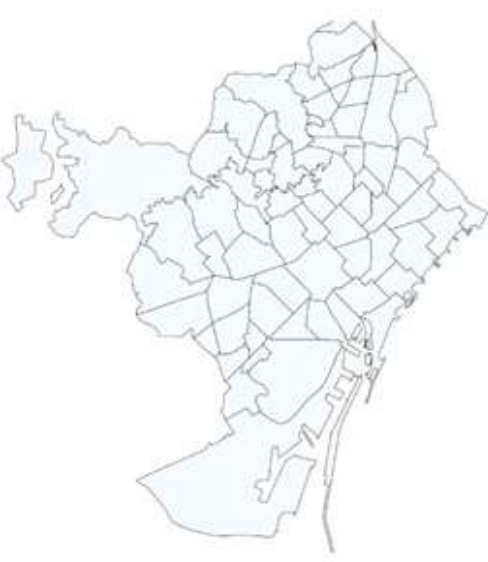

Scenario 1

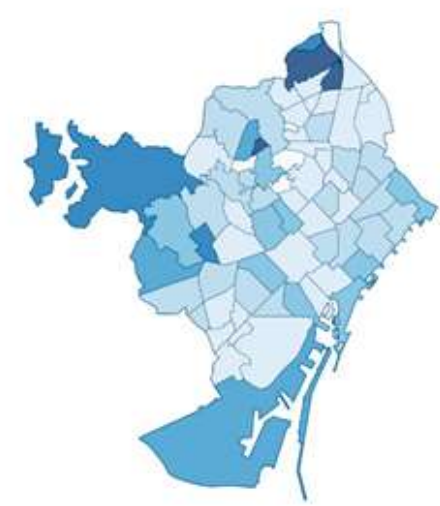

Scenario 3 


\subsection{The case of New York City}

Using our UDSS model (table 3), we apply it for the case of Manhattan in New York city. COVID-19 has largely spread in New York City with concerning increases in community transmission in certain neighbourhoods like Manhattan[1]. We categorize all the SMBs according to the 4 clusters. We discuss this assumption of generalization in the section 6.3. The visual results are shown in the figure 6 . In a pre-quarantine and standard scenario, the average SMBs occupancy of Manhattan was $83 \%$. In scenario 1 , the average SMBs occupancy is $26 \%$. In the scenario 2 , the average SMBs occupancy is $82 \%$. In the scenario 3 , the average SMBs occupancy is $69 \%$.

Figure 6: Visualization of the different scenarios of Manhattan close-down
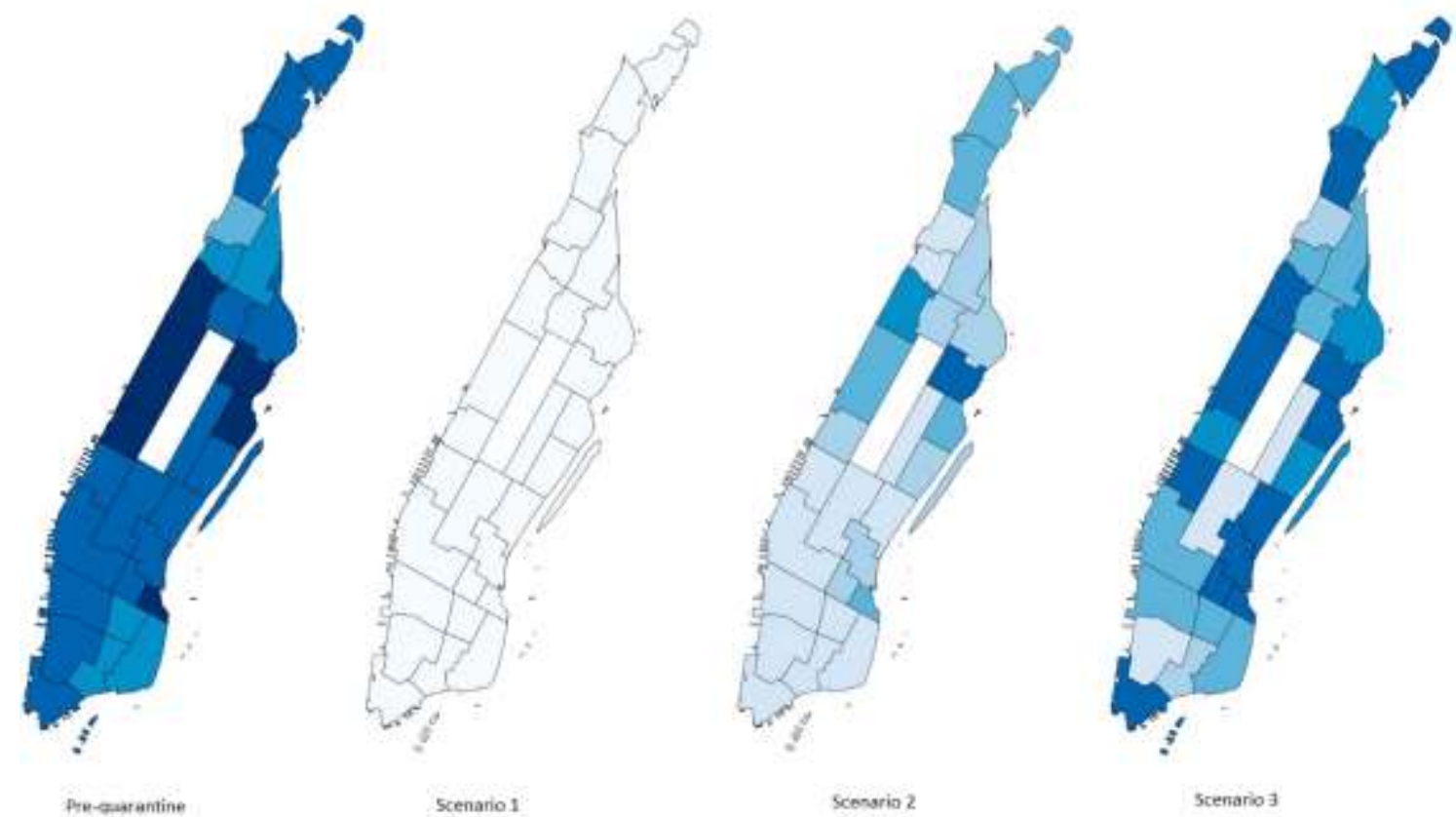

\section{Discussion}

This study identifies new varieties and sources of information (geographical, epidemiologist, opinions of users) and leverages this mix of varieties to manage better uncertainty during a global pandemic. It compares 3 classes of $\mathrm{Al}$ algorithms and determines the best one in 
terms of effectiveness to classify locations. The recent pandemic crisis has shown that governments need help to abandon the improvisation of their actions and obtain more datadriven insights in order to take more robust decisions. Based on 3 possible scenarios, the UDSS supports the actions of the government by calculating and visualizing their impact in terms of SMBs closure.

\subsection{Implications for practitioners}

After 76 days of lockdown, the central Chinese city of Wuhan, the initial epicentre of the COVID-19 outbreak, reopened its doors allowing planes, trains and cars to leave the city. Their success was due to a clear strategy of the government, composed of three important factors: (a) a massive testing which provided a great deal of medical data, (b) the ban of inter-city transports and (c) the closure of the non-essential SMBs in the infected territory. But these actions were possible due to the extensive collaboration and cooperation inside the region between public and private workforces. Collaboration: the non-essential activities were stopped while the essential ones were able to keep going with more social distancing in public spaces or at work [39]. Cooperation: the most affected areas were isolated, but the distribution of food and medicine were covered by the less affected ones [40].

In turn, European countries had no strategy to fight this pandemic even if a lot of specialists had warned of its imminence. A study [41] calculated that between 1980 and 2010 the number of epidemic outbreaks of infectious diseases had tripled. In Spain, many decisions between February and June 2020 were contradictory from a day to another ${ }^{3}$. The UDSS may improve collaboration and cooperation and create a model which is similar to that of China.

\footnotetext{
${ }^{3}$ https://elpais.com/ciencia/2020-04-23/no-teniamos-un-plan-de-que-hacer-en-una-pandemia.html last access: 29/11/2020
} 
Collaboration: by having a forecast of their actions on the local economy, the governments could be more transparent, efficient and so better understood. Consequently, the citizens could collaborate more and took precautions more seriously (for instance, at the weekend many citizens left town and went to their second residence to avoid confinement and as a result spread the virus outside their community).

Cooperation: working with the UDSS results, governments could identify zones of neighbourhoods that were less affected than others in terms of SMBs closure. They could ask then cooperation of SMBs between neighbourhoods, to avoid shops running out of stock and ensure all citizens are able to access to essential products or services $[13,40]$.

The UDSS also has an application for the Post-COVID period. Indeed, many governments have recently announced stimulus packages to bolster industry and services and recover from the economic catastrophe of the pandemic [7]. These programmes include a massive cash injection for SMBs at risk of bankruptcy along with the creation of more hospitals and the recruitment of more medical staff. Today, governments are struggling to find the perfect criteria to allocate these subventions by sectors and they are receiving a lot of pressure from lobbying activists (Retail, Culture, Sport...). Our study could avoid possible clientelism by calculating the real number of SMBs closures and offering a criterion to calculate this necessary stimulus package.

\subsection{Implications for research}

Our research shows the possible combination of different sources of information (geographical, epidemiologist, user survey) to develop a decision-making system to manage uncertainty during a global pandemic. The evidence that Al can be employed along with traditional tools in the Health and Public sectors is fairly recent. IBM report that $90 \%$ of all the data in the world has been generated over the last two years. Data creates better 
knowledge and great progress is being made in many industries to resolve unanswered management issues [42]. However, the health sector is a conservative industry which is still a little behind when it comes to deploying and implementing such techniques. This study helps to cover part of this gap along with others (e.g.[8]).

In March 2020, the coronavirus sounded as an alarm which led to consumers hysterically rushing out to purchase household goods such as toilet paper or pasta. This hoarding behaviour is explained as a loss of control for the consumer largely aggravated by the disruptions of international supply chains and the lack of assortment of products in the supermarkets [13]. This reaction shows the actions decided by the government had deep emotional and irrational effects on the population. They were largely diffused and commented in the social channels like Twitter and can be detected using semantic tool analysis $[8,10]$. The UDSS establishes responses to scenarios but further lines of investigation should propose Al-based solutions to collect the reaction of the citizens, in other words, the grade of acceptation or rejection. This information should be included as another input for the UDSS.

\subsection{Limitations and generalizability}

As with all the modelling approaches, our model is based on 4 predictors. We present hereunder their limitations and so possible lines of improvement. First, the financial risk of a location is related to two conditions (geo-localization and number of workers by square meters). We know it is an estimation since other variables could be included (cash-flow situation, price of the product, renting costs etc.). Second, the COVID contagion risk level is based on different surveys carried out in May and June 2020 when little information about the virus was known[23]. Consequently, the responses between the epidemiologists were 
not homogeneous and similar surveys should be reiterated in the future to corroborate our results.

Third, we know the habits of the customers have changed forever [17]. During the pandemic, many consumers have turned to Do-It-Yourself (DIY) projects and home-made activities such as baking or cooking becoming players in the "markerspace"[15]. The question remains if the old habits will die or return in the Post COVID new reality. The consulting company Ernst \& Young [6] states that two-thirds of consumers say their future purchase decisions will be strongly influenced by how companies have responded and helped society during the pandemic. $33 \%$ of the interviewees affirm that they have switched from a brand to another because they have perceived an unfortunate response to the pandemic. Pent-up demand is a familiar consequence when access to market is denied for a period of time [17]. Its qualitative analysis makes understand what the citizens' preferences or new priorities are in a Post-COVID reality. Its quantitative examination allows to seize the possible rebound in consumption and its impact on the national GDP [17]. In the objective to extend our results, we believe that the user preferences survey should be regularly done to take into account possible changes in consumer habits and to measure the evolution of the pent-up demand.

In this study, the authors have used the UDSS results of Barcelona for the case of the Manhattan neighbourhood of New York City. This assumption is legitimated by recent studies which show the globalization has generated the uniformization and standardisation of urban services offer [43-46]. The (hidden) objective is that the visitors feel at home wherever they are travelling as they can find their preferred brands all over the world. In fact, the observatory Eixos is able to categorize SMBs from different cities of the planet using the same list of business categories. 
Besides, the proposed model was designed for COVID-19 but it could be generalized and extended to other pandemic outbreaks: Malaria, avian flu or SARS. The adaption would deal with the disease transmission risk feature and the epidemiologists' recommendations survey $(f 3(p)$ and $f 4(p)$ variables).

\section{Conclusion}

Taking the right decision to cope with a sanitary alert which, in the highest period of the outbreak, infects thousands of people every day and substantially jeopardizes the economic situation of a whole country, requires the most efficient Decision Support System available. Prior to this research, there was no decision-aid system capable of providing a holistic evaluation of the consequences for urban SMBs. This study fills this gap by providing an Albased and non-traditional DSS.

The DSS combines new varieties and sources of information (geographical, epidemiologist, user opinions). It has various capabilities which have been strongly demonstrated with the two case studies of Barcelona and New York City. The Al algorithm (k-Means) that is finally used to compute the model, is based on a benchmarking of 3 different classes of clustering. 4 different predictors are considered and the results obtained reveal potential gains for governments: a better prediction should improve the management of the public and private workforces, the optimization of the recovery financial packages and a better understanding for the citizens of the actions taken by the government.

Further lines of research could be necessary for the evolution of the proposed model such as updating the users' preferences votes or the improvement of the epidemiologists' analysis. Other studies could look also to include new features, such as the degree of acceptance by citizenship. 


\section{Acknowledgement}

The authors acknowledge research assistance from the Eixos Observatory team located at Barcelona (Spain) (https://eixos.cat/html/portada-1-eng.html ). They gratefully acknowledge the support of the two co-Founders: David Nogué and Carlos Carrasco during the data collection and analysis and visualization phases.

\section{List of Abbreviations}

COVID: 'CO' stands for corona, 'VI' for virus, and 'D' for disease

SMBs: Small and Medium Businesses

DSS : Decision Support System

SARS : Severe Acute Respiratory Syndrome

BIRCH: Balanced Iterative Reducing and Clustering using Hierarchies

DBSCAN: Density-based spatial clustering of applications with noise

\section{Authors' contribution}

All the authors have made equivalent contribution to the conception, analysis of the work and have drafted the work and substantively revised it. All authors read and approved the final manuscript.

\section{Funding}

Not applicable. 


\section{Availability of data and materials}

The datasets used and/or analysed during the current study are available from the corresponding author on reasonable request.

\section{Competing interests}

The authors declare that they have no competing interests.

\section{Appendix A: User preferences survey}

Figure A1: Instructions (translated in English)

The DS4DS research group from Ramon Llull University - La Salle is participating in the fight against the COVID-19 pandemic, which is why it has developed this survey that contains 30 questions.

Many types of businesses and institutions have been closed in order to slow the spread of the coronavirus. This survey is intended to help government departments make decisions about the de-escalation process.

Select from the two options proposed, the one that has more importance of reopening according to your criteria and regardless of whether they are already open or not. The total response time to all questions is between 5 to 10 minutes.

At the end of this survey, you can leave your email to receive the conclusions of the analysis. https://qfreeaccountssic1.az1.qualtrics.com/ife/form/SV 9Rk5hLoFuMHVIDT

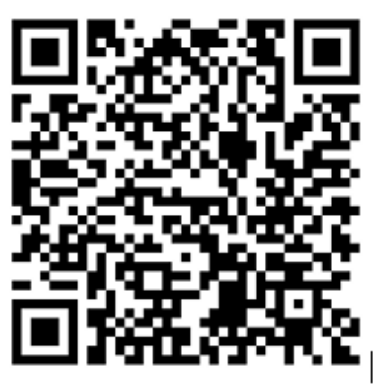


Figure A2: Sampling of the questionnaire (translated in English):

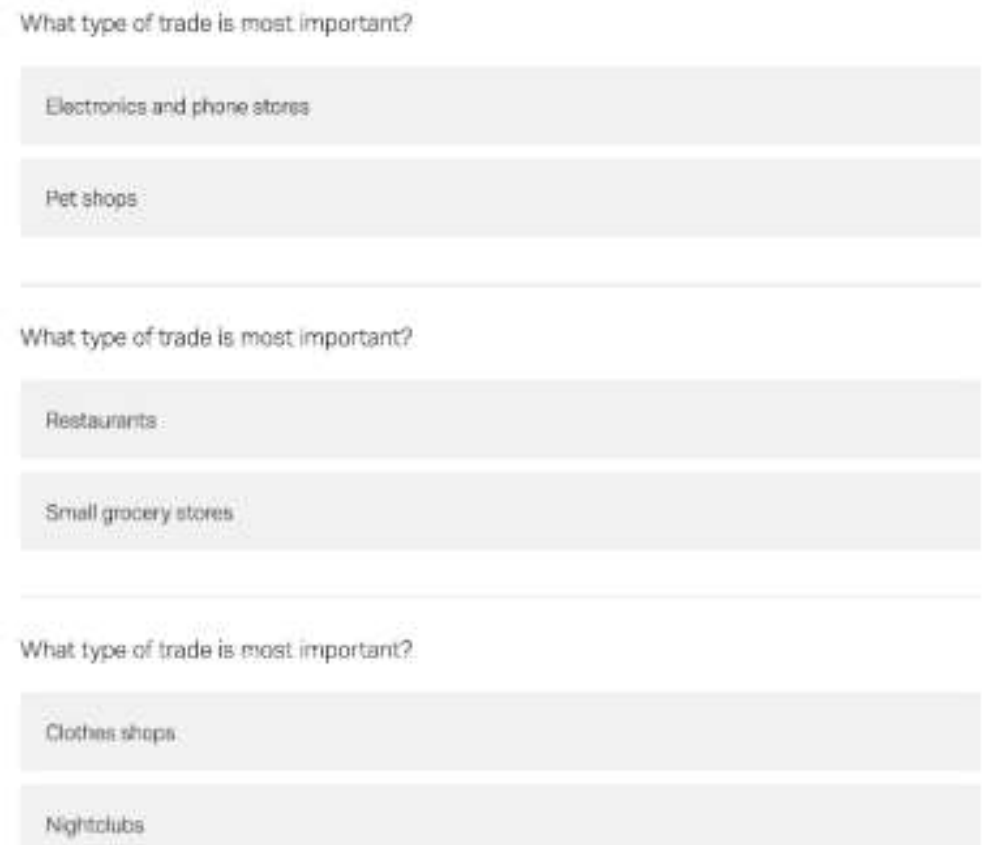

Figure A3: Age distribution of the respondents

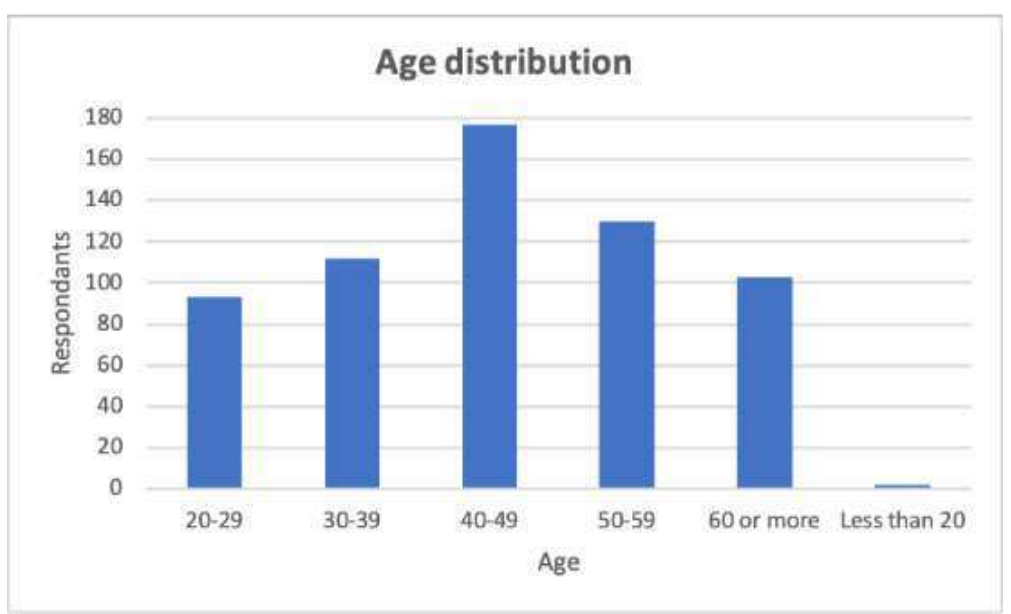

\section{Declaration}

Ethics approval and consent to participate

Not applicable

Consent for publication

Not applicable 


\section{References}

[1] J.H. Center, Johns Hopkins Coronavirus Resource Center, (2020). https://coronavirus.jhu.edu/map.html last access on 30/11/2020 (accessed December 3, 2020).

[2] S. Barua, Understanding Coronanomics: The Economic Implications of the Coronavirus (COVID-19) Pandemic, SSRN Electron. J. (2020) 1-44. https://doi.org/10.2139/ssrn.3566477.

[3] R.A. Medina, 1918 Influenza Virus: 100 Years on, Are We Prepared Against the Next Influenza Pandemic?, Nat. Rev. Microbiol. 16 (2018) 61-62.

https://doi.org/10.1038/nrmicro.2017.174.

[4] N. Fernandes, Economic effects of coronavirus outbreak ( COVID-19) on the world economy Nuno Fernandes Full Professor of Finance IESE Business School Spain, SSRN Electron. Journal, ISSN 1556-5068, Elsevier BV,. (2020) 0-29.

[5] N. Caroll, COVID-19'S IMPACT ON THE UK GROCERY MARKET, (2020). https://www.mintel.com/blog/retail-market-news/covid-19s-impact-on-the-ukgrocery-market (accessed December 3, 2020).

[6] Ernst\&Young, COVID-19 enterprise resilience, (2020). https://www.ey.com/en_gl/covid-19 (accessed December 3, 2020).

[7] McKinsey, The next normal, (2020). https://www.mckinsey.com/featuredinsights/coronavirus-leading-through-the-crisis (accessed December 3, 2020).

[8] J. Ginsberg, M.H. Mohebbi, R.S. Patel, L. Brammer, M.S. Smolinski, L. Brilliant, Detecting influenza epidemics using search engine query data, Nature. 457 (2009) 1012-1014. https://doi.org/10.1038/nature07634.

[9] M.S. Gerber, Predicting crime using Twitter and kernel density estimation, Decis. Support Syst. 61 (2014) 115-125. https://doi.org/10.1016/j.dss.2014.02.003.

[10] D. Grimaldi, Can we analyse political discourse using Twitter ? Evidence from Spanish 2019 presidential election, Soc. Netw. Anal. Min. (2019) 1-9. https://doi.org/10.1007/s13278-019-0594-6.

[11] C.W. Potter, A history of influenza, J. Appl. Microbiol. (2001) 572-579.

[12] M. Mueller-Peltzer, S. Feuerriegel, A. Molgaard Nielsen, A. Kongsted, W. Vach, D. Neumann, Longitudinal healthcare analytics for disease management: Empirical demonstration for low back pain, Decis. Support Syst. 132 (2020) 113271. https://doi.org/10.1016/j.dss.2020.113271.

[13] A. Sharma, A. Adhikary, S.B. Borah, Covid-19's impact on supply chain decisions: Strategic insights from NASDAQ 100 firms using Twitter data, J. Bus. Res. 117 (2020) 443-449. https://doi.org/10.1016/j.jbusres.2020.05.035.

[14] P. Sharma, T.Y. Leung, R.P.J. Kingshott, N.S. Davcik, S. Cardinali, Managing uncertainty during a global pandemic: An international business perspective, J. Bus. Res. 116 (2020) 188-192. https://doi.org/10.1016/j.jbusres.2020.05.026.

[15] E. Pantano, G. Pizzi, D. Scarpi, C. Dennis, Competing during a pandemic? Retailers' ups and downs during the COVID-19 outbreak, J. Bus. Res. 116 (2020) 209-213. 
https://doi.org/10.1016/j.jbusres.2020.05.036.

[16] M. Sigala, Tourism and COVID-19: Impacts and implications for advancing and resetting industry and research, J. Bus. Res. 117 (2020) 312-321.

https://doi.org/10.1016/j.jbusres.2020.06.015.

[17] J. Sheth, Impact of Covid-19 on consumer behavior: Will the old habits return or die?, J. Bus. Res. 117 (2020) 280-283. https://doi.org/10.1016/j.jbusres.2020.05.059.

[18] F. Eggers, Masters of disasters? Challenges and opportunities for SMEs in times of crisis, J. Bus. Res. 116 (2020) 199-208. https://doi.org/10.1016/j.jbusres.2020.05.025.

[19] Y. Lu, J. Wu, J. Peng, L. Lu, The perceived impact of the Covid-19 epidemic: evidence from a sample of 4807 SMEs in Sichuan Province, China, Environ. Hazards. 19 (2020) 323-340. https://doi.org/10.1080/17477891.2020.1763902.

[20] A.L. Kristof, Person-Organization fit: an integrative review of its conceptualizations, measurement, and implications, BMJ Open. 9 (2019).

https://doi.org/10.1136/bmjopen-2018-026266.

[21] D. Nogué, C. Carrasco, Eixos Report, Barcelona Act. (2016). http://barcelonaoberta.cat/wp-content/uploads/2017/06/informe-eixos-definitiu.pdf (accessed December 10, 2020).

[22] R.T. Carson, T. Groves, Incentive and informational properties of preference questions, Environ. Resour. Econ. 37 (2007) 181-210. https://doi.org/10.1007/s10640-007-9124-5.

[23] M. Sanger-Katz, C.C. Miller, Q. Bui, When 511 Epidemiologists Expect to Fly, Hug and Do 18 Other Everyday Activities Again, New York Times. (2020) 6.

https://www.nytimes.com/interactive/2020/06/08/upshot/when-epidemiologists-willdo-everyday-things-coronavirus.html.

[24] B. Pietsch, The risk levels of everyday activities like dining out, going to the gym, and getting a haircut, according to an infectious-disease expert, Bus. Insid. (2020). https://www.businessinsider.com/riskiest-to-least-risky-activities-during-coronaviruspandemic-ranked-2020-5?r=US\&IR=T\#low-risk-outdoor-activities-11.

[25] Staff, Explainer: The coronavirus risks of everyday activities as economies reopen, Reuters. (2020). https://uk.reuters.com/article/uk-health-coronavirus-risksexplainer/explainer-the-coronavirus-risks-of-everyday-activities-as-economies-reopenidUKKBN23N1UG.

[26] T. McLean, These are the riskiest activities to do during the coronavirus pandemic, SF Gate. (2020). https://www.sfgate.com/bayarea/slideshow/These-are-the-riskiestactivities-to-do-during-203341.php.

[27] A. AUBREY, L. WAMSLEY, C. WROTH, From Camping To Dining Out: Here's How Experts Rate The Risks Of 14 Summer Activities, NPR. (2020). https://www.npr.org/sections/health-shots/2020/05/23/861325631/from-camping-todining-out-heres-how-experts-rate-the-risks-of-14-summeractivit? $\mathrm{t}=1606734542752 \& \mathrm{t}=1606821916247$.

[28] D. Byrne, What Is Complexity Science?, 3 (2001) 61-76.

[29] P.C. Fiss, A SET-THEORETIC APPROACH TO ORGANIZATIONAL CONFIGURATIONS, Acad. Manag. Rev. 32 (2007) 1180-1198. https://doi.org/10.3109/10253890.2015.1121984.

[30] R.H. Samet, Complexity, the science of cities and long-range futures, Futures. 47 (2013) 
49-58. https://doi.org/10.1016/j.futures.2013.01.006.

[31] T. DesOrmeau, From hair salons to gyms, experts rank 36 activities by coronavirus risk level, Mass Live. (2020). https://www.masslive.com/coronavirus/2020/06/from-hairsalons-to-gyms-experts-rank-36-activities-by-coronavirus-risklevel.html?fbclid=IwAROCcM2xGXe6jJt6xPjqo4laL11E3ePqAfcynAkCPWa_rNQ48kSzAL0 COGQ.

[32] J. MacQUEEN, SOME METHODS FOR CLASSIFICATION AND ANALYSIS OF MULTIVARIATE OBSERVATIONS, 1967. https://doi.org/10.1007/s11665-016-2173-6.

[33] T. Zhang, R. Ramakrishnan, M. Livny, BIRCH: An Efficient Data Clustering Method for Very Large Databases, in: Proc. 1996 ACM SIGMOD Int. Conf. Manag. Data, Association for Computing Machinery, New York, NY, USA, 1996: pp. 103-114. https://doi.org/10.1145/233269.233324.

[34] H.D. Wijaya, S. Dwiasnati, Clustering of Earthquake Data Using Kohonen Self Organizing Maps (SOMs) Algorithm, 9771 (2019) 384-388. https://doi.org/10.21276/sb.2019.5.7.11.

[35] M. Ester, H.-P. Kriegel, J. Sander, X. Xu, A density-based algorithm for discovering clusters in large spatial databases with noise, in: AAAI Press, 1996: pp. 226-231.

[36] D.J. KETCHEN, C.L. SHOOK, THE APPLICATION OF CLUSTER ANALYSIS IN STRATEGIC MANAGEMENT RESEARCH: AN ANALYSIS AND CRITIQUE, Strateg. Manag. J. 17 (1996) 441-458. https://doi.org/https://doi.org/10.1002/(SICI)10970266(199606)17:6<441::AID-SMJ819>3.0.CO;2-G.

[37] Y. Liu, Z. Li, H. Xiong, X. Gao, J. Wu, Understanding of internal clustering validation measures, Proc. - IEEE Int. Conf. Data Mining, ICDM. (2010) 911-916. https://doi.org/10.1109/ICDM.2010.35.

[38] F. Pedregosa, G. Varoquaux, A. Gramfort, V. Michel, B. Thirion, O. Grisel, M. Blondel, P. Prettenhofer, R. Weiss, V. Dubourg, J. Vanderplas, A. Passos, D. Cournapeau, M. Brucher, M. Perrot, É. Duchesnay, Scikit-learn: Machine learning in Python, J. Mach. Learn. Res. 12 (2011) 2825-2830.

[39] D. Cyranoski, What China'S Coronavirus the Rest of the World, Nature. 579 (2020) 479480. https://media.nature.com/original/magazine-assets/d41586-020-00741-x/d41586020-00741-x.pdf.

[40] E. Han, M.M.J. Tan, E. Turk, D. Sridhar, G.M. Leung, K. Shibuya, N. Asgari, J. Oh, A.L. García-Basteiro, J. Hanefeld, A.R. Cook, L.Y. Hsu, Y.Y. Teo, D. Heymann, H. Clark, M. McKee, H. Legido-Quigley, Lessons learnt from easing COVID-19 restrictions: an analysis of countries and regions in Asia Pacific and Europe, Lancet. 0 (2020) 1-10. https://doi.org/10.1016/S0140-6736(20)32007-9.

[41] K.F. Smith, M. Goldberg, S. Rosenthal, L. Carlson, J. Chen, C. Chen, S. Ramachandran, Global rise in human infectious disease outbreaks, J. R. Soc. Interface. 11 (2014) 1-6. https://doi.org/10.1098/rsif.2014.0950.

[42] D.J. Pauleen, W.Y.C. Wang, Does big data mean big knowledge? KM perspectives on big data and analytics, J. Knowl. Manag. 21 (2017) 1-6. https://doi.org/10.1108/JKM-082016-0339.

[43] G. Ritzer, E.L. Malone, S.A. Studies, T. End, G. Ritzer, E.L. Malone, Globalization Theory : Lessons from the Exportation of McDonaldization and the New Means of Consumption 
Published by : Mid-America American Studies Association Globalization Theory : Lessons from the Exportation of McDonaldization and the New Means of Con, 41 (2016) 97-118.

[44] G. Ritzer, P. Dean, N. Jurgenson, The Coming of Age of the Prosumer, (2012). https://doi.org/10.1177/0002764211429368.

[45] S. Seran, P. Monica, Prosumer engagement in innovation strategies The Prosumer Creativity and Focus Model, (2014). https://doi.org/10.1108/MD-06-2013-0347.

[46] D. Grimaldi, V. Fernandez, C. Carrasco, Heuristic for the localization of new shops based on business and social criteria, Technol. Forecast. Soc. Change. (2018) 1-9. https://doi.org/10.1016/j.techfore.2018.07.034. 